\title{
Influence of Muscle-Tendon Wrapping on Calculations of Joint Reaction Forces in the Equine Distal Forelimb
}

\author{
Jonathan S. Merritt, ${ }^{1}$ Helen M. S. Davies, ${ }^{2}$ Colin Burvill,, ${ }^{1}$ and Marcus G. Pandy ${ }^{1}$ \\ ${ }^{1}$ Department of Mechanical and Manufacturing Engineering, Melbourne School of Engineering, The University of Melbourne, \\ VIC 3010, Australia \\ ${ }^{2}$ Department of Veterinary Clinic and Hospital, Faculty of Veterinary Science, The University of Melbourne, \\ VIC 3030, Australia \\ Correspondence should be addressed to Jonathan S. Merritt, merritt@unimelb.edu.au
}

Received 14 September 2007; Accepted 24 December 2007

Recommended by Daniel Howard

The equine distal forelimb is a common location of injuries related to mechanical overload. In this study, a two-dimensional model of the musculoskeletal system of the region was developed and applied to kinematic and kinetic data from walking and trotting horses. The forces in major tendons and joint reaction forces were calculated. The components of the joint reaction forces caused by wrapping of tendons around sesamoid bones were found to be of similar magnitude to the reaction forces between the long bones at each joint. This finding highlighted the importance of taking into account muscle-tendon wrapping when evaluating joint loading in the equine distal forelimb.

Copyright () 2008 Jonathan S. Merritt et al. This is an open access article distributed under the Creative Commons Attribution License, which permits unrestricted use, distribution, and reproduction in any medium, provided the original work is properly cited.

\section{INTRODUCTION}

Mechanical loads experienced by the coffin (distal interphalangeal) and fetlock (metacarpophalangeal) joints of horses are thought to be related to injuries that occur in these joints. Mechanical stresses are thought to play a role in cartilage wear [1] and are likely to be the cause of many changes to subchondral bone that are associated with lameness [2] Tendon wrapping is also thought to relate to the mechanics of injuries. For example, the influence of the deep digital flexor tendon (DDFT) on navicular disease $[3,4]$ and the relationship between interosseous ligament (IL) injury and metacarpal injury [5] are both presumed to be mediated by the forces generated when the two tendinous structures wrap around underlying bones. Consequently, knowledge of joint loads is likely to be relevant in understanding the mechanical pathogenesis of many kinds of injury in horses.

Little is currently known about the joint reaction forces of the equine distal forelimb. The wrapping of the DDFT about the distal sesamoid (navicular) bone has been investigated in very few studies $[3,4,6-8]$. Biewener et al. [9] calculated reaction forces at the coffin and fetlock joints, but those authors did not take into account forces produced by wrapping of the tendons around the sesamoid bones. Thomason [10] studied the architecture of the distal third metacarpal bone and estimated approximate values for the force between the proximal sesamoid bones and the distal metacarpus, but he did not perform any calculations based upon a dynamic model of the limb.

The aim of this study was to calculate and report the major joint reaction force components in the fetlock and coffin joints during walking and trotting in the horse. The forces exerted by wrapping of the tendons around both the proximal and distal sesamoid bones were taken into account when performing the joint reaction force calculations. The main hypothesis was that the forces exerted due to tendon wrapping would contribute substantially to the net reaction forces at the joints and hence could not be neglected.

\section{MATERIALS AND METHODS}

\subsection{Kinetic and kinematic data}

Three Quarter Horses (Equus caballus) with masses of $500 \mathrm{~kg}, 545 \mathrm{~kg}$, and $500 \mathrm{~kg}$ were used for the study. Three retroreflective markers were attached to the skin over bony 
TABLE 1: Parameters for the distal forelimb model. The $x$ axis is directed from the proximal joint of the given segment toward the distal joint and the $y$ axis is directed cranially. $x$ and $y$ values, defining either the origin and insertion points of muscle-tendon paths, or the centers of pulleys and via points, are given as percentages of their respective segment lengths.

\begin{tabular}{|c|c|c|c|c|c|}
\hline Tendon & Element & Segment & Radius (mm) & $x(\%)$ & $y(\%)$ \\
\hline \multirow{3}{*}{ SDFT } & Pulley & Metacarpus & 40.1 & 3.4 & 7.2 \\
\hline & Via Point & Pastern & - & -7.1 & -27.6 \\
\hline & Insertion & Pastern & - & 74.5 & -13.9 \\
\hline \multirow{3}{*}{ IL } & Origin & Metacarpus & - & 21.6 & 0.0 \\
\hline & Pulley & Pastern & 19.3 & 0.0 & 0.0 \\
\hline & Insertion & Pastern & - & 74.5 & -13.9 \\
\hline \multirow{4}{*}{ DDFT } & Origin & Metacarpus & 40.1 & 3.4 & 7.2 \\
\hline & Via Point & Pastern & - & 1.6 & -31.0 \\
\hline & Pulley & Hoof & 15.0 & 3.4 & -8.9 \\
\hline & Insertion & Hoof & - & 32.0 & -11.6 \\
\hline
\end{tabular}

parts of each segment of the right forelimb, where the segments were defined as the hoof, pastern, metacarpus, antebrachium, and brachium. Rather than being separated into portions corresponding to the first and second phalanges, the pastern was treated as a single rigid body because the proximal interphalangeal joint was previously described as approximately rigid [11]. Additional markers were then placed over the centers of the joints. A Peak Performance Motus system was used to measure the locations of the markers during a static trial, in which the horse stood quietly and approximately square.

The joint center markers were removed, and the horses were led in hand over a Bertec 4000 series force plate at the walk and trot. The ground reaction force was measured by the force plate, while kinematic data were simultaneously collected from the three markers of each limb segment. The horses were led so that one of the three orthogonal axes of the laboratory coordinate system corresponded to the direction of progression, and the other two axes were then presumed to be part of a parasagittal plane. A minimum of two trials and a maximum of four were collected for each horse at each gait, resulting in a total of 9 walking trials and 11 trotting trials. Marker position data were acquired at a frequency of $60 \mathrm{~Hz}$, while ground reaction force data were acquired at $600 \mathrm{~Hz}$.

\subsection{Joint moment calculation}

Kinematic and ground reaction force data from the walking and trotting trials were filtered using lowpass, forwardreverse Butterworth filters with mirror-symmetric boundary conditions. A cutoff frequency of $5 \mathrm{~Hz}$ was used for the kinematic data at the walk, while $12 \mathrm{~Hz}$ was used for the trot. The ground reaction force was filtered using a cutoff frequency of $12 \mathrm{~Hz}$ at both gaits. After filtering, the kinematic data were upsampled using cubic splines to a frequency of $600 \mathrm{~Hz}$ in order to match the sampling frequency of the ground reaction force. Noise was removed from the marker positions of the static trials by calculating the mean marker positions for each trial.
The joint center locations were calculated for the walking and trotting trials by referencing their locations during the static trial of each horse. The three markers of each limb segment that were common to both the static trial and the locomotion trials were used to calculate the joint center locations using three-dimensional rigid body transformations. For each sample of the locomotion trials, a transformation was calculated for each limb segment that moved the position of the joint center marker in the static trial to its expected position in the locomotion trial, under the assumption that the limb segment was a rigid body [12]. The center of each joint was calculated as the average of the positions predicted by the segment proximal to the joint and the segment distal to the joint. The two-dimensional, sagittal plane joint angles of the limb during each locomotion trial were then calculated after projecting the joint center locations to the sagittal plane.

The sagittal plane joint moments [13] were calculated for the stance phase of each locomotion trial, defined as the portion of the trial during which the vertical ground reaction force was greater than $50 \mathrm{~N}$. The joint moments were calculated using two different methods: standard inverse dynamics and a massless, quasistatic analysis. In the first method, the inertial parameters (mass and mass moment of inertia) of each limb segment were approximated using regression equations determined for a set of Dutch Warmblood horses [14]. For the second method, both the gravitational mass and inertial parameters of each segment were set equal to zero.

\subsection{Forelimb model}

A two-dimensional model of the distal forelimb was developed, based upon the model described by Meershoek et al. [15]. Brown et al. [16] performed tendon excursion measurements to find the moment arms of several muscles in the equine distal forelimb about the carpus and fetlock joints. The moment arm values measured by Brown et al. [16] were substantially different from those of the model described by Meershoek et al. [15], and because of this discrepancy, the model was modified so that its moment arms matched 
those reported by Brown et al. [16] Figure 1 illustrates the modified forelimb model, while Table 1 describes the complete model geometry. A comparison of the moment arms of the superficial digital flexor tendon(SDFT) and DDFT at the fetlock joint, as reported by both Meershoek et al. [15] and Brown et al. [16], yielded a scaling factor of 0.68. All pulley radii in the Meershoek et al. [15] model were scaled by this value.

Uniform scaling of pulley radii did not yield a good match at the fetlock for the variation in moment arm with joint angle as reported by Brown et al. [16]. In order to more correctly simulate this variation, the pulleys of the SDFT and DDFT were replaced by a single via point for each tendon, whose location represented a point of wrapping around the proximal sesamoid bones. The paired proximal sesamoid bones are connected to the first phalanx by their straight, oblique, and short distal ligaments [17]. These relatively short, strong ligaments are unlikely to experience considerable length changes during locomotion [10], and hence the wrapping locations of the tendons around the fetlock joint were approximated as fixed relative to the pastern. The locations of the via points were chosen to correspond to the approximate anatomical locations of the sesamoid bones, and were then fine-tuned to produce moment arms which closely matched those reported by Brown et al. [16]. The comparison between model moment arms and the values published by Brown et al. [16] is shown in Figure 2.

\subsection{Tendon tension and joint reaction force calculations}

The forelimb model was used to calculate the tensions in the SDFT, DDFT, and IL, as well as the reaction forces at the coffin and fetlock joints. Following the method described by Meershoek et al. [15], the strain in the IL was first calculated assuming that the rest length of this ligament was its length at the start of the stance phase [15]. The tension in the IL was calculated using an experimentally-derived force-length relationship [15]. The tension of the DDFT was then calculated by balancing the joint moment at the coffin joint with the moment generated by this tendon. A similar calculation was performed at the fetlock joint, to calculate the tension in the SDFT, after subtracting the moments exerted about this joint by both the IL and the DDFT.

Finally, joint reaction forces were calculated at the coffin and fetlock joints by enforcing static equilibrium of all forces acting on the segments shown in Figure 3. The DDFT wrapped around the distal sesamoid (navicular) bone, causing it to be compressed against the distal palmar articular surface of the second phalanx [4]. In a similar way, the SDFT, DDFT, and IL wrapped around the proximal sesamoid bones, causing them to be compressed against the palmar surface of the distal metacarpal condyles. These wrapping forces were considered as part of the calculation of joint reaction forces.

The duration of the stance phase of each trial varied slightly. Consequently, after all quantities had been calculated, the stance phase duration was normalized to a

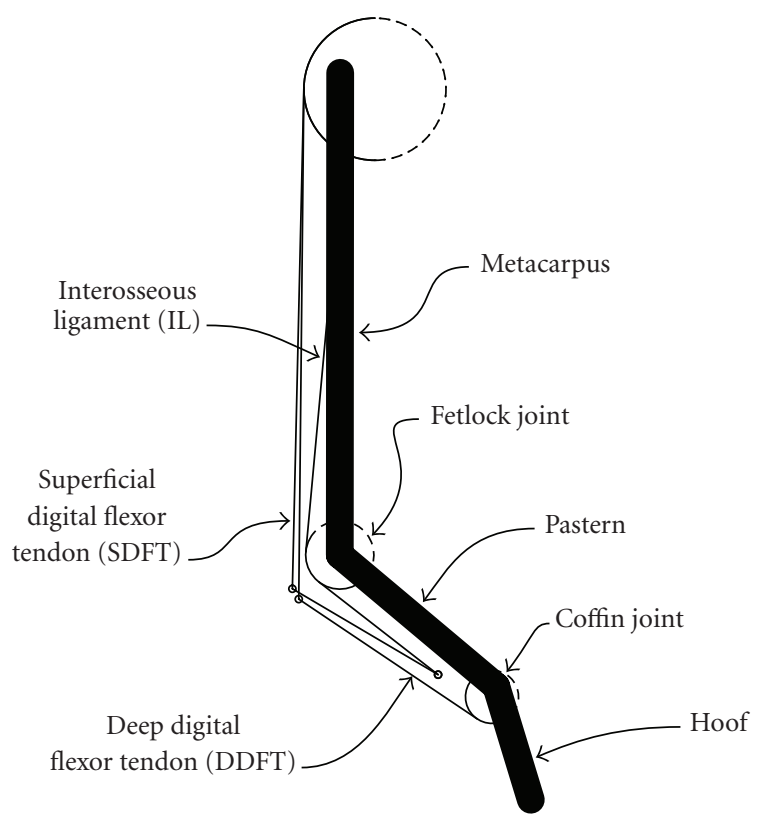

FIgure 1: Two-dimensional, sagittal plane model of the equine forelimb. The model contained three rigid body segments and three major tendinous structures.

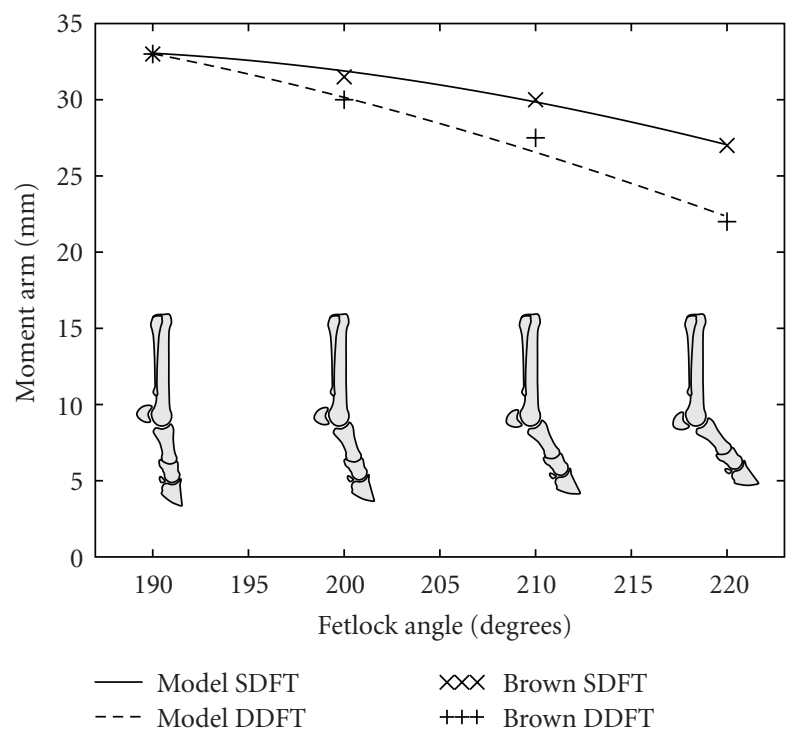

Figure 2: Moment arms from the forelimb model compared with those published by Brown et al. [16]. Moment arms for the forelimb model were calculated using a simulated tendon excursion method.

percentage scale, with $0 \%$ representing the first hoof-ground contact and $100 \%$ representing the last hoof-ground contact. The time sample of each trial was scaled linearly to fit this range. Following this, the results were scaled by body mass, by dividing them by the mass of each horse expressed in kilograms. The results were then averaged over all trials to obtain estimates of the mean and standard deviation of each quantity. Due to the small number of trials available, all trials 


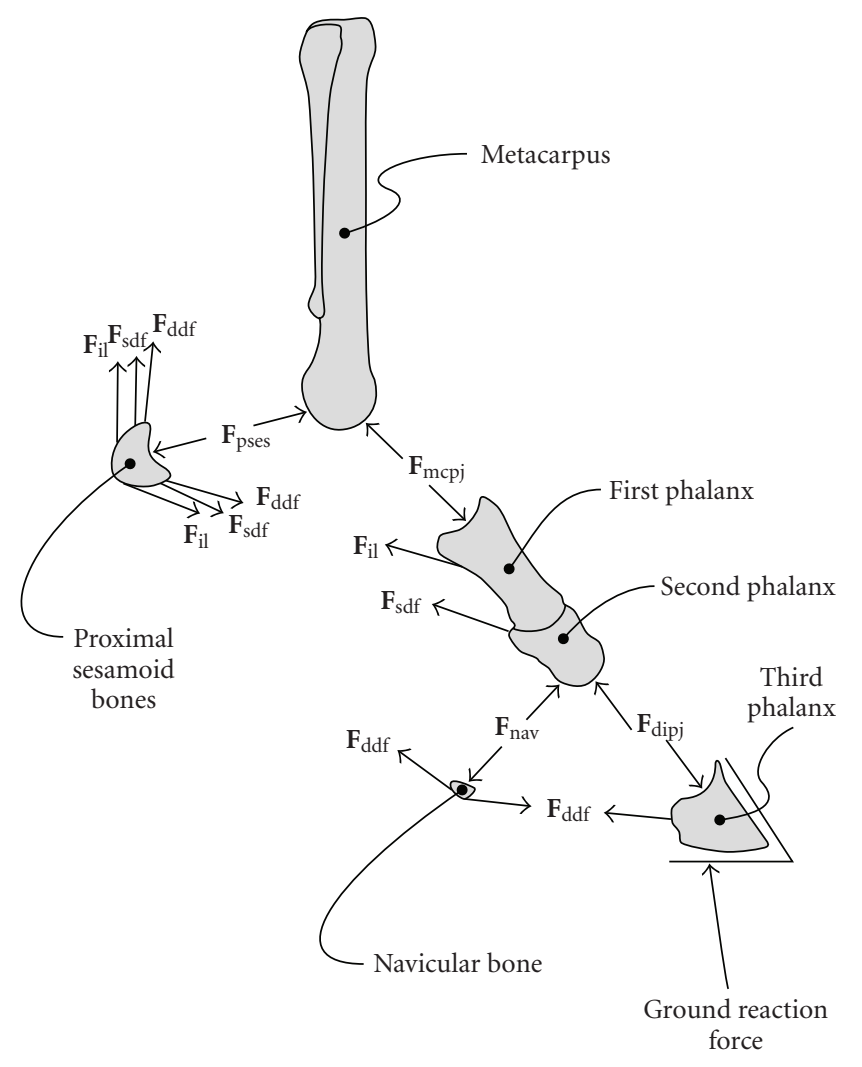

FIGURE 3: Forces considered when calculating the joint reaction forces at the fetlock and coffin joints. The forces were the ground reaction force, the force exerted by the distal phalanx on the middle phalanx $\left(\mathbf{F}_{\text {dipj }}\right)$, the force exerted by the navicular bone on the middle phalanx $\left(\mathbf{F}_{\text {nav }}\right)$, the DDFT force $\left(\mathbf{F}_{\mathrm{ddf}}\right)$, the SDFT force $\left(\mathbf{F}_{\mathrm{sdf}}\right)$, the IL force $\left(F_{i 1}\right)$, the force exerted by the proximal phalanx on the distal metacarpus $\left(\mathbf{F}_{\mathrm{mcpj}}\right)$, and the force exerted by the proximal sesamoid bones on the distal metacarpus $\left(\mathbf{F}_{\text {pses }}\right)$.

were weighted equally in this calculation, despite the fact that some horses performed more trials than others.

\section{RESULTS AND DISCUSSION}

The model presented in this paper had several important limitations. The two-dimensional nature of the model limited it to considering only those force components which could be projected onto the sagittal plane. This approximation may affect all of the calculated quantities, especially where forces are oriented so as to be partially oblique to a parasagittal plane. Additionally, frontal asymmetry of the limb, both in its loading and anatomy, may influence the internal loads significantly. The importance of frontal plane asymmetry is suggested by many clinical phenomena; for example, the greater reported occurrence of proximal sesamoid bone fracture in the medial bone compared with the lateral one in racing Thoroughbreds [18]. The model presented in this paper presumed perfect frontal symmetry, and hence would be unable to predict the effects of different loading or anatomy of the medial and lateral sides of the limb.
Only the major anatomical structures of the distal limb were modeled. In reality, the proximal sesamoid bones are not fixed relative to the pastern, and additional structures such as the distal sesamoidean ligaments, the collateral ligaments of the joints, and the extensor branches of the suspensory (interosseous) ligament are all likely to exert loads on the bones, which would change the estimates of joint loading obtained in this study. The decision to include only the major structures of the limb was motivated both by a desire for simplicity in the model and a lack of data regarding the morphology and mechanical properties of the remaining structures. The justification for this decision lies mainly in precedent, for example, [15], and the anatomical observation that the structures that were included in the model were physically much larger than those which were neglected.

The exact zero strain (rest) length of IL of the model was unknown, and so it was approximated as the length of the IL during early stance, following the method described by Meershoek and Lanovaz [19]. It was observed that in the model, the IL behaved as a nonlinear angular spring, where the moment it exerted about the fetlock was dependent only upon the fetlock angle. Hence, variations in the rest length of IL would affect the relative sharing of the moment at the fetlock joint between the IL and SDFT. It would be beneficial for a future study to investigate directly the relationship between the IL moment about the fetlock and the fetlock joint angle, rather than inferring the moment about this joint from angle-strain and strain-force relationships.

The model simulated the moment arms of the SDFT and DDFT at the fetlock that were reported by Brown et al. [16]. These moment arms were measured by the tendon excursion method, and were substantially different from those reported previously in the literature $[9,15,20]$. The reason for the discrepancy in moment arm values is not known, but may arise from different measurement methodologies or different breeds of horse being used. Further investigation would be beneficial to the development of mechanical models of the equine forelimb in the future. Variation in the SDFT and DDFT moment arms at the fetlock could affect the calculated tension in the SDFT.

Figure 4 shows the joint moments calculated at the coffin, fetlock, carpus, and elbow joints for walking and trotting. The calculated joint moments were comparable with those reported previously in the literature [21, 22]. These two previous studies $[21,22]$ used Dutch Warmblood horses, whereas the current study used Quarter Horses. The difference in horse breeds may account for small differences in the joint moments.

In Figure 4, it is evident that the massless, quasistatic calculation of joint moments produced results very similar to the full inverse dynamics calculation for all joints. To the best knowledge of the authors, the direct comparison of these two techniques for calculating joint moments in the equine distal forelimb has never been reported, despite the reliance on quasistatic solutions for several previous distal forelimb studies, for example, [23]. The relative importance of the inertial and mass-related components of the moments increased for the more proximal joints of the limb, where greater masses and greater accelerations were involved. The 

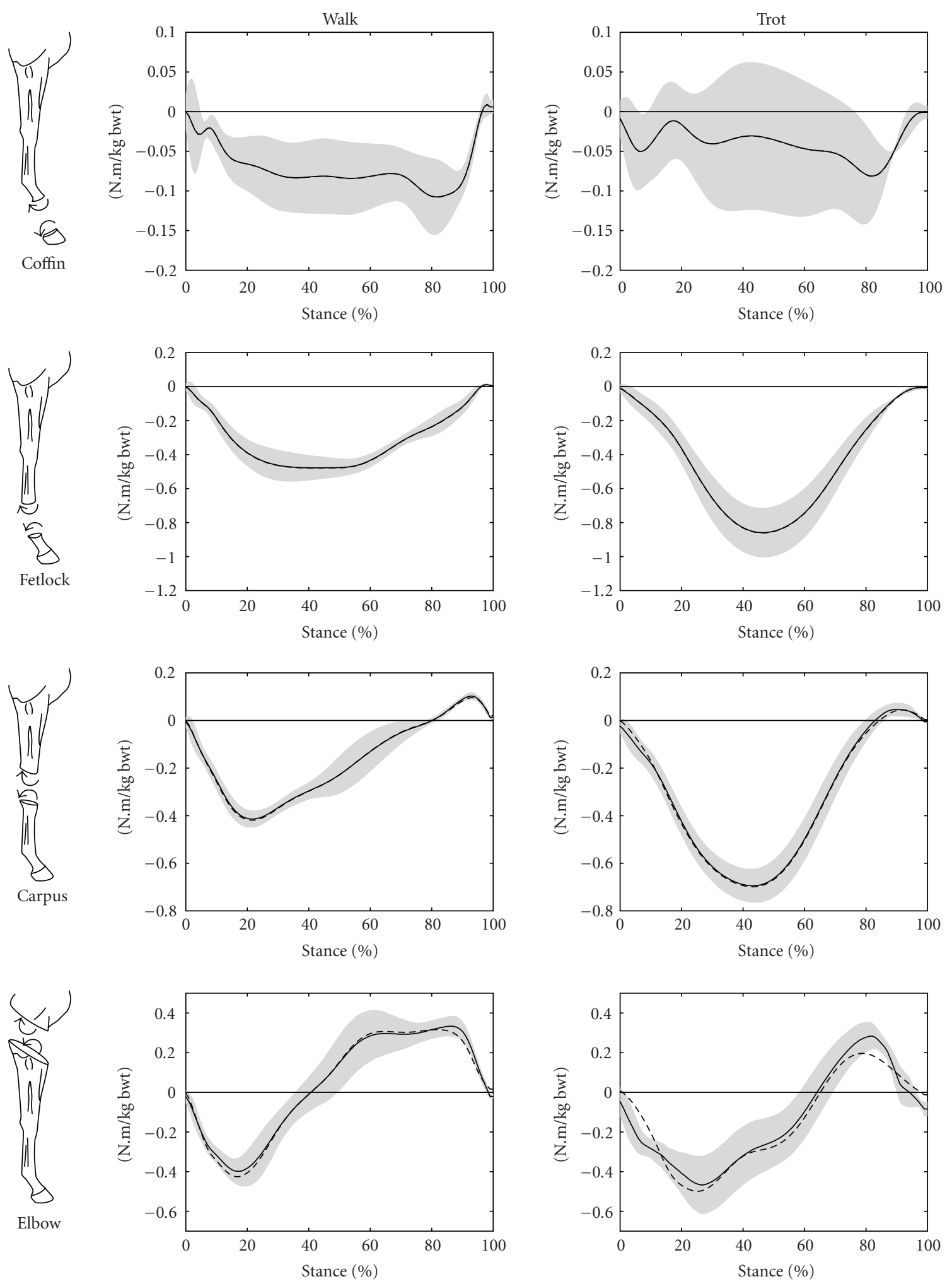

_ Inverse dynamics solution

- - Quasi-static solution

_ Inverse dynamics solution
- - Quasi-static solution

FIGURE 4: Joint moments calculated at the coffin, fetlock, carpus, and elbow joints for walking and trotting during the stance phase of the stride. The mean values of the inverse dynamics solutions are shown as solid lines with grey shading representing \pm 1 SD, while the mean values of the massless, quasi-static analysis are shown as dashed lines. The stance phase of the stride was the time during which the vertical component of the ground reaction force was greater than 50 N. $0 \%$ of stride was the time of first hoof-ground contact, while $100 \%$ of stride was the time of last hoof-ground contact. 


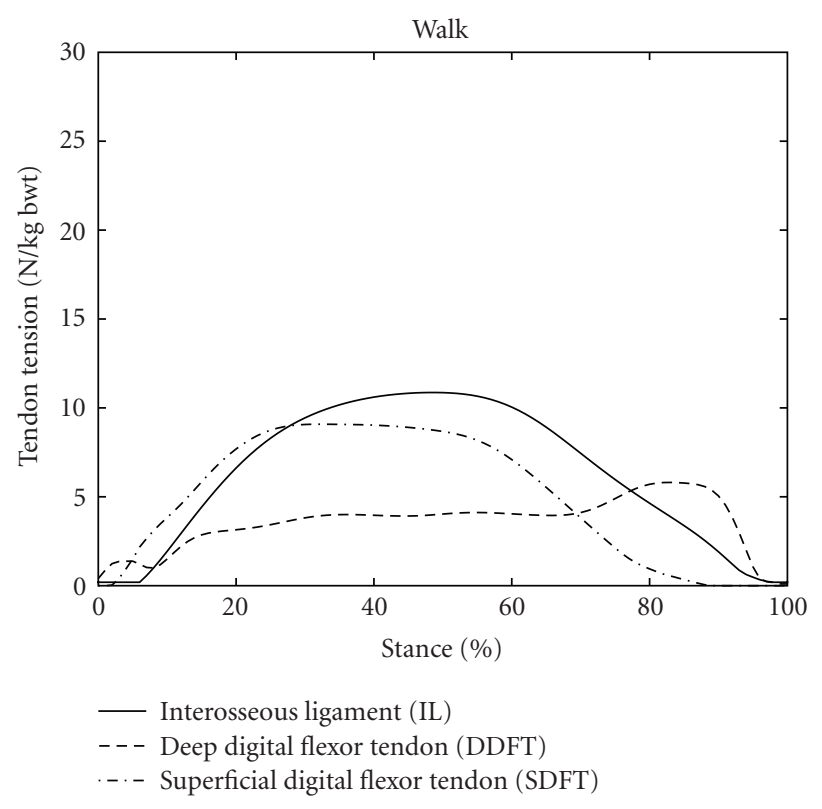

(a)

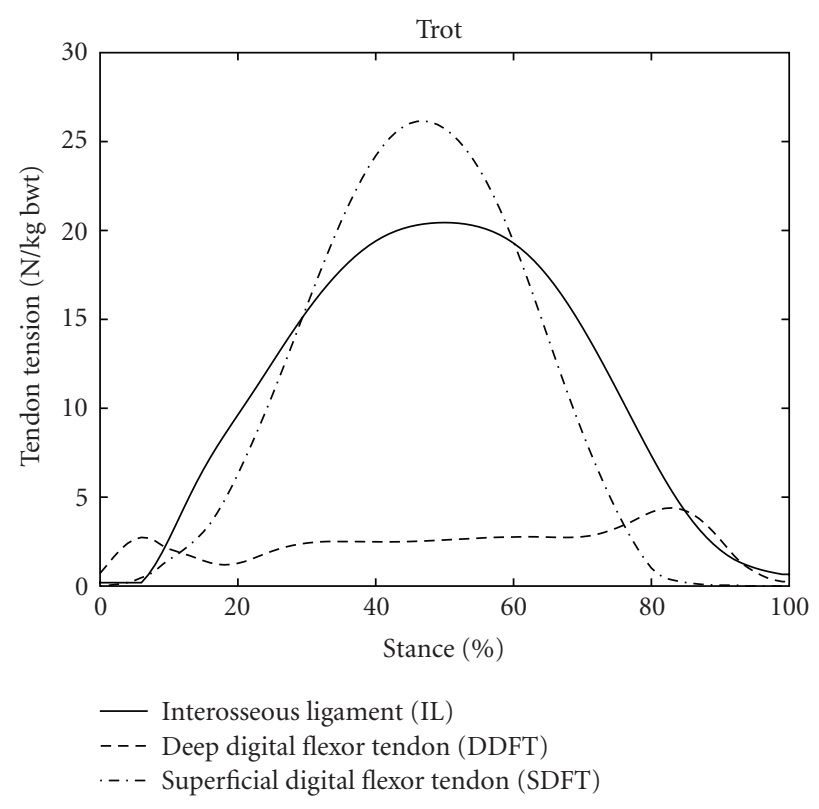

(b)

FIGURE 5: Mean tendon tensions in the DDFT, SDFT, and IL for walking and trotting during the stance phase of the stride. The stance phase of the stride was the time during which the vertical component of the ground reaction force was greater than $50 \mathrm{~N}$. $0 \%$ of stride was the time of first hoof-ground contact, while $100 \%$ of stride was the time of last hoof-ground contact.

insensitivity of the results to inertial factors during the stance phase contrasted with the reported importance of these factors during the swing phase [24]. This contrast may readily be understood as a result of the ground reaction force, which dominated the joint moment calculations during the stance phase, yet is absent by definition during the swing phase. In the present model, each forelimb distal to the carpus accounted for only $0.6 \%$ of body mass [14], and this region of the limb experienced little acceleration during stance. Hence it was unlikely that any inertial or mass-related effects from this region of the limb would have a significant effect on calculated joint moments or other aspects of limb dynamics.

The mean tensions in the DDFT, SDFT, and IL are shown in Figure 5 for walking and trotting. The tendon tensions during walking were similar to those calculated by Jansen et al. [25], from in vivo tendon strain measurements. The tensions during trotting were somewhat different from those previously reported by Meershoek and Lanovaz [19], but the differences may be accounted for by the substantially different moment arms of the tendons at the fetlock in the two studies. In the present study, the tendon moment arms at the fetlock were configured to simulate values determined experimentally using the tendon excursion method [16], while those used by Meershoek and Lanovaz [19] were obtained using a geometric method [15]. The rest length of IL was also unknown in the present study, and so it was approximated using the method described by Meershoek and Lanovaz [19]. Variations in the IL rest length would cause changes in the IL and SDFT tensions.

Magnitudes of the components of the joint reaction forces are shown in Figure 6 for walking and trotting. Shown are the force exerted by the distal phalanx on the middle phalanx, the force exerted by the navicular bone on the middle phalanx, the force exerted by the proximal phalanx on the distal metacarpus, and the force exerted by the paired proximal sesamoid bones on the distal metacarpus.

In a previous report of joint reaction forces in the equine distal forelimb, Biewener et al. [9] noted that forces may be generated from tendon wrapping, but these authors did not calculate those forces and ignored them in their subsequent analysis of bone strains. Thomason [10] estimated the magnitude of the wrapping force at the proximal sesamoid bones from assumptions derived from studies of the architecture of the distal third metacarpal bone, but he did not perform any calculations based upon a dynamic model of the limb. Several previous studies $[3,4,6-8]$ calculated the wrapping force of the DDFT about the distal sesamoid bone, and a link has been postulated between this force and navicular disease. However, to the best knowledge of the authors, the remaining joint reaction results represent novel data which have not previously been reported.

The calculated force exerted by the navicular bone on the middle phalanx was similar to that reported previously $[3,4,6-8]$. In particular, this force exhibited a peak in late stance, which was related to the change in the point of application (point of zero moment) of the ground reaction force [4]. It was observed that as the hoof neared breakover (the point in the stride at which the heels of the hoof depart from the ground and the hoof "break over" the toe), the point of application of the ground reaction force moved from a location within the body of the hoof to a point at the toe, which increased the moment arm of this force about the coffin joint. During late stance, this increase in moment arm 


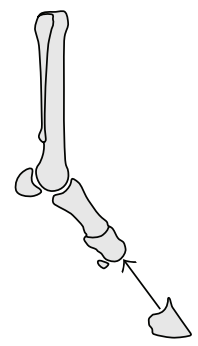

Distal phalanx

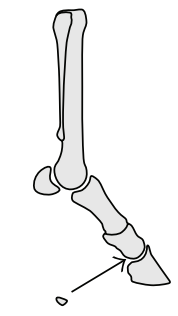
Navicular bone

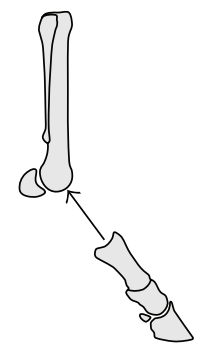

Proximal phalanx

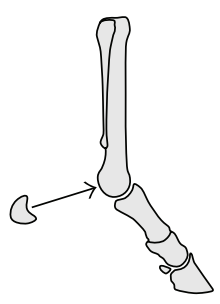

Proximal sesamoid bones
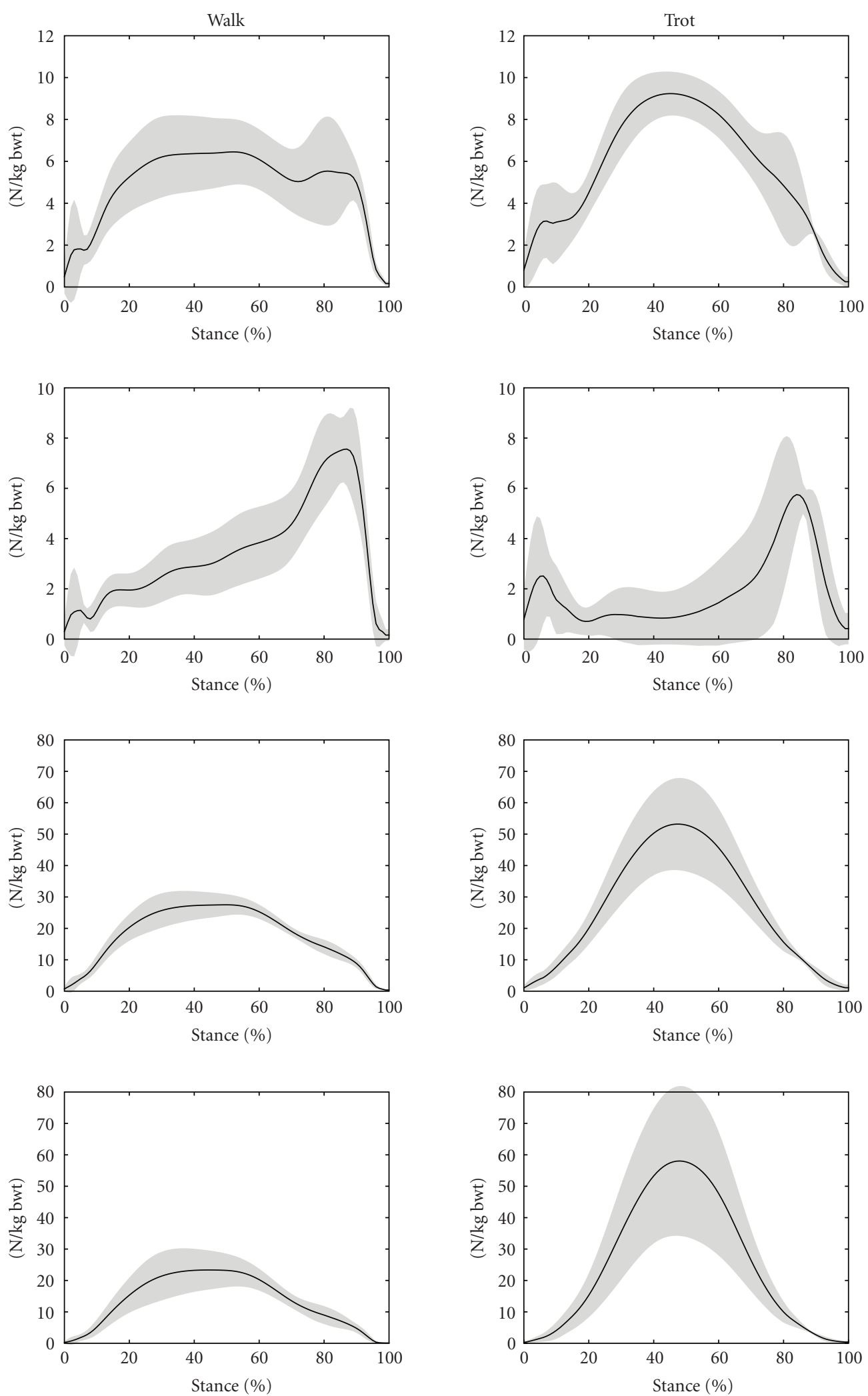

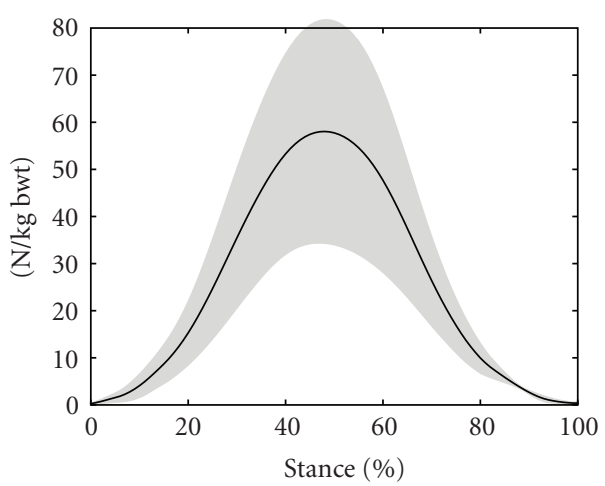

Figure 6: Magnitudes of calculated joint reaction force components from long bones and sesamoid bones in the coffin and fetlock joints for walking and trotting during the stance phase of the stride. The magnitudes are shown as solid lines with grey shading to represent $\pm 1 \mathrm{SD}$. The stance phase of the stride was the time during which the vertical component of the ground reaction force was greater than $50 \mathrm{~N}$. $0 \%$ of stride was the time of first hoof-ground contact, while $100 \%$ of stride was the time of last hoof-ground contact. 
was adequate to raise the value of the coffin joint moment (Figure 4, Coffin), DDFT tension (Figure 5, DDFT), and the force applied by the navicular bone (Figure 6, Navicular Bone) despite a decrease in the magnitude of the ground reaction force in late stance relative to midstance (observed in the ground reaction force data, but not shown here). The increase in DDFT tension in late stance did not produce such an obvious peak in the reaction force between the distal and middle phalanges (Figure 6, Distal Phalanx) although a bimodal pattern was evident in this component during walking (Figure 6, Distal Phalanx, Walk).

The central hypothesis of this paper-that the forces induced by wrapping of tendons around the sesamoid bones are important components of the net joint reaction forces of the distal forelimb-is well illustrated in Figure 6. In the top two rows of Figure 6, the magnitude of the distal phalanx force and that of the navicular bone are both comparable, as are the magnitudes of the proximal phalanx and proximal sesamoid bone forces shown in the bottom two rows. Hence, the magnitudes of forces exerted by the sesamoid bones of the joints were comparable to those exerted between the long bones themselves. Neglect of either of the two forces from the sesamoid bones would cause a substantial change in the calculated net joint reaction force.

Knowledge of the reaction forces at the joints is potentially important for future biomechanical studies into areas such as the behavior of joint cartilage, subchondral bone loading, and bone remodeling. Future studies are expected to involve three-dimensional finite-element modeling of the mechanical interactions at the joints. The model presented in this paper, and its calculated joint reaction forces, could be used as a reference point for the predictions of more sophisticated models.

\section{CONCLUSIONS}

Joint reaction forces in the equine distal forelimb were calculated using a two-dimensional mathematical model. Components of these forces that were generated by wrapping of tendons about the sesamoid bones in the limb were found to be of similar magnitude to those generated between the long bones of each limb segment. These wrapping forces serve to illustrate the importance of the sesamoid bones in the mechanical function of the forelimb, and also provide insight into the possible mechanical cause of numerous injuries related to loading of the joints.

\section{ACKNOWLEDGMENTS}

The authors would like to thank Drs Nick Brown, Chris Kawcak, and C. Wayne McIllwraith for the use of the kinematic and kinetic data from three Quarter Horses. These data were collected using facilities at Colorado State University.

\section{REFERENCES}

[1] R. M. Bowker, P. J. Atkinson, T. S. Atkinson, and R. C. Haut, "Effect of contact stress in bones of the distal interphalangeal joint on microscopic changes in articular cartilage and ligaments," American Journal of Veterinary Research, vol. 62, no. 3, pp. 414-424, 2001.

[2] C. E. Kawcak, C. W. McIlwraith, R. W. Norrdin, R. D. Park, and P. S. Steyn, "Clinical effects of exercise on subchondral bone of carpal and metacarpophalangeal joints in horses," American Journal of Veterinary Research, vol. 61, no. 10, pp. 1252-1258, 2000.

[3] M. P. McGuigan and A. M. Wilson, "The effect of bilateral palmar digital nerve analgesia on the compressive force experienced by the navicular bone in horses with navicular disease," Equine Veterinary Journal, vol. 33, no. 2, pp. 166-171, 2001.

[4] A. M. Wilson, M. P. McGuigan, L. Fouracre, and L. MacMahon, "The force and contact stress on the navicular bone during trot locomotion in sound horses and horses with navicular disease," Equine Veterinary Journal, vol. 33, no. 2, pp. 159-165, 2001.

[5] S. S. Le Jeune, M. H. Macdonald, S. M. Stover, K. T. Taylor, and M. Gerdes, "Biomechanical investigation of the association between suspensory ligament injury and lateral condylar fracture in thoroughbred racehorses," Veterinary Surgery, vol. 32, no. 6, pp. 585-597, 2003.

[6] E. Eliashar, M. P. McGuigan, K. A. Rogers, and A. M. Wilson, "A comparison of three horseshoeing styles on the kinetics of breakover in sound horses," Equine Veterinary Journal, vol. 34, no. 2, pp. 184-190, 2002.

[7] E. Eliashar, M. P. McGuigan, and A. M. Wilson, "Relationship of foot conformation and force applied to the navicular bone of sound horses at the trot," Equine Veterinary Journal, vol. 36, no. 5, pp. 431-435, 2004.

[8] M. A. Willemen, H. H. C. M. Savelberg, and A. Barneveld, "The effect of orthopaedic shoeing on the force exerted by the deep digital flexor tendon on the navicular bone in horses," Equine Veterinary Journal, vol. 31, no. 1, pp. 25-30, 1999.

[9] A. A. Biewener, J. Thomason, A. Goodship, and L. E. Lanyon, "Bone stress in the horse forelimb during locomotion at different gaits: a comparison of two experimental methods," Journal of Biomechanics, vol. 16, no. 8, pp. 565-576, 1983.

[10] J. J. Thomason, "The relationship of structure to mechanical function in the third metacarpal bone of the horse, Equus caballus," Canadian Journal of Zoology, vol. 63, no. 6, pp. 14201428, 1985.

[11] C. Degueurce, H. Chateau, H. Jerbi, et al., "Three-dimensional kinematics of the proximal interphalangeal joint: effects of raising the heels or the toe," Equine Veterinary Journal. Supplement, no. 33, pp. 79-83, 2001.

[12] J. H. Challis, "A procedure for determining rigid body transformation parameters," Journal of Biomechanics, vol. 28, no. 6, pp. 733-737, 1995.

[13] W. Back and H. Clayton, Eds., Equine Locomotion, W.B. Saunders, Philadelphia, Pa, USA, 2000.

[14] H. H. F. Buchner, H. H. C. M. Savelberg, H. C. Schamhardt, and A. Barneveld, "Inertial properties of Dutch Warmblood horses," Journal of Biomechanics, vol. 30, no. 6, pp. 653-658, 1997.

[15] L. S. Meershoek, A. J. van den Bogert, and H. C. Schamhardt, "Model formulation and determination of in vitro parameters of a noninvasive method to calculate flexor tendon forces in the equine forelimb," American Journal of Veterinary Research, vol. 62, no. 10, pp. 1585-1593, 2001.

[16] N. A. T. Brown, M. G. Pandy, W. L. Buford, C. E. Kawcak, and C. W. McIlwraith, "Moment arms about the carpal and metacarpophalangeal joints for flexor and extensor muscles 
in equine forelimbs," American Journal of Veterinary Research, vol. 64, no. 3, pp. 351-357, 2003.

[17] K. M. Dyce, W. O. Sack, and C. J. G. Wensing, Textbook of Veterinary Anatomy, W.B. Saunders, Philadelphia, Pa, USA, 1987.

[18] E. J. Parente, D. W. Richardson, and P. Spencer, "Basal sesamoidean fractures in horses: 57 cases (1980-1991)," Journal of the American Veterinary Medical Association, vol. 202, no. 8, pp. 1293-1297, 1993.

[19] L. S. Meershoek and J. L. Lanovaz, "Sensitivity analysis and application to trotting of a noninvasive method to calculate flexor tendon forces in the equine forelimb," American Journal of Veterinary Research, vol. 62, no. 10, pp. 1594-1598, 2001.

[20] A. J. van den Bogert, "Computer simulation of locomotion in the horse," Ph.D. thesis, The University of Utrecht, Utrecht, The Netherlands, 1989.

[21] H. M. Clayton, J. L. Lanovaz, H. C. Schamhardt, M. A. Willemen, and G. R. Colborne, "Net joint moments and powers in the equine forelimb during the stance phase of the trot," Equine Veterinary Journal, vol. 30, no. 5, pp. 384-389, 1998.

[22] G. R. Colborne, J. L. Lanovaz, E. J. Sprigings, H. C. Schamhardt, and H. M. Clayton, "Forelimb joint moments and power during the walking stance phase of horses," American Journal of Veterinary Research, vol. 59, no. 5, pp. 609-614, 1998.

[23] H. F. Schryver, D. L. Bartel, N. Langrana, and J. E. Lowe, "Locomotion in the horse: kinematics and external and internal forces in the normal equine digit in the walk and trot," American Journal of Veterinary Research, vol. 39, no. 11, pp. 1728-1733, 1978.

[24] J. L. Lanovaz and H. M. Clayton, "Sensitivity of forelimb swing phase inverse dynamics to inertial parameter errors," Equine Veterinary Journal. Supplement, no. 33, pp. 27-31, 2001.

[25] M. O. Jansen, A. J. van den Bogert, D. J. Riemersma, and H. C. Schamhardt, "In vivo tendon forces in the forelimb of ponies at the walk, validated by ground reaction force measurements," Acta Anatomica, vol. 146, no. 2-3, pp. 162167, 1993. 

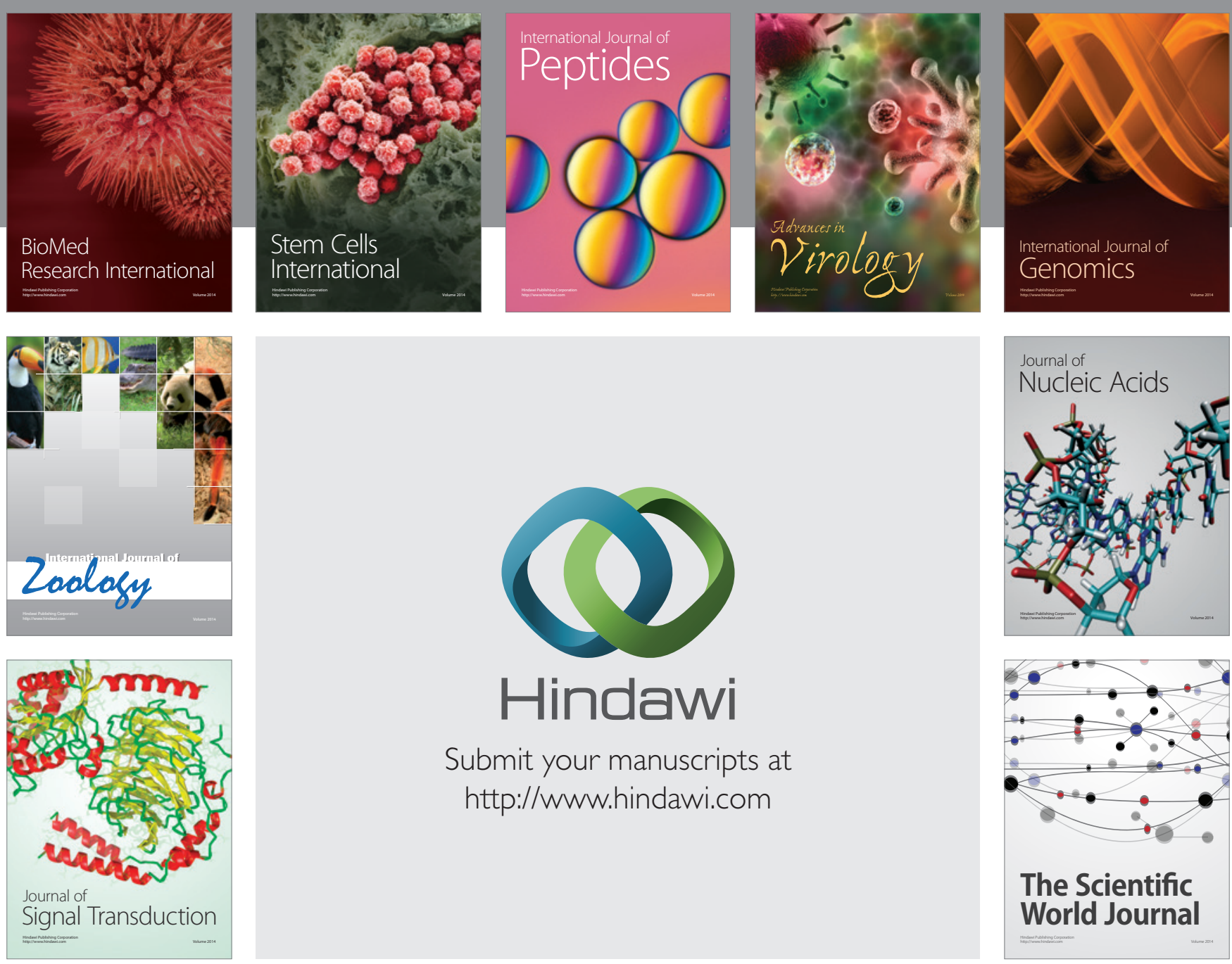

Submit your manuscripts at

http://www.hindawi.com
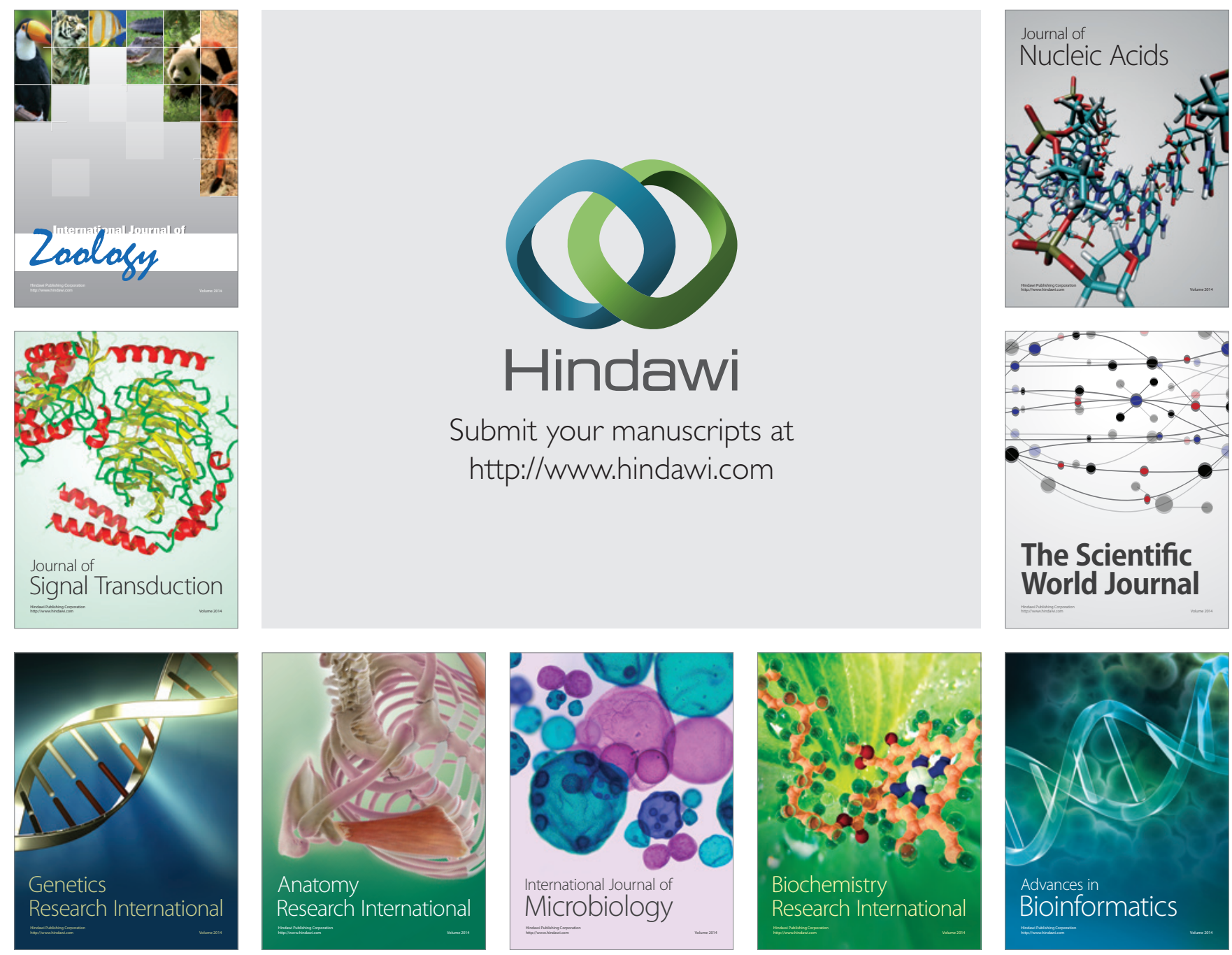

The Scientific World Journal
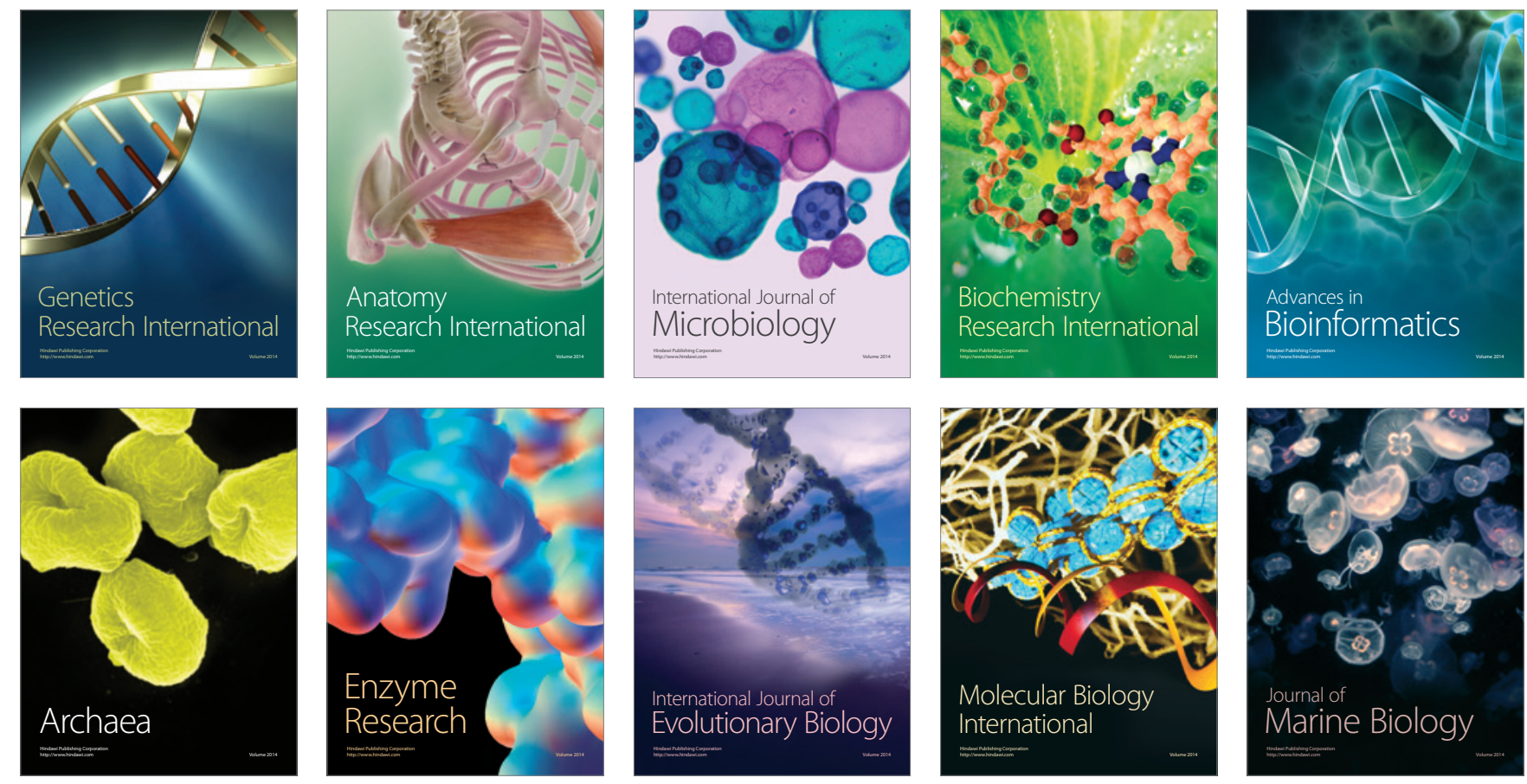